\title{
Process reconstruction from incomplete and/or inconsistent data
}

\author{
Mário Ziman ${ }^{1,2}$, Martin Plesch ${ }^{1}$, Vladimír Bužek ${ }^{1,2}$ \\ ${ }^{1}$ Research Center for Quantum Information, Slovak Academy of Sciences, Dúbravská cesta 9, 84511 Bratislava, Slovakia \\ ${ }^{2}$ Faculty of Informatics, Masaryk University, Botanická 68a, 602 00 Brno, Czech Republic
}

\begin{abstract}
We analyze how an action of a qubit channel (map) can be estimated from the measured data that are incomplete or even inconsistent. That is, we consider situations when measurement statistics is insufficient to determine consistent probability distributions. As a consequence either the estimation (reconstruction) of the channel completely fails or it results in an unphysical channel (i.e., the corresponding map is not completely positive). We present a regularization procedure that allows us to derive physically reasonable estimates (approximations) of quantum channels. We illustrate our procedure on specific examples and we show that the procedure can be also used for a derivation of optimal approximations of operations that are forbidden by the laws of quantum mechanics (e.g., the universal NOT gate).
\end{abstract}

\section{INTRODUCTION}

For any reliable quantum information processing it is important to know how states of quantum systems are transformed under the action of quantum channels (maps). It is therefore essential to develop tools by means of which we can acquire knowledge about properties of quantum channels. Providing we have no prior knowledge about an action of a particular transformation our task is to determine characteristics of a corresponding quantum map based on correlations between input and out states of quantum systems that serve as probes of the channel.

In principle, the action of a quantum channel can be probed in two different ways: (1) The first option is to use as an input a single entangled state of a bi-partite system [1-4]. One particle (e.g., a qubit) of this bi-partite system is transformed under the action of the channel while the second particle remains unchanged (or evolves according to a known transformation). By performing a complete quantum tomography of the bi-partite system at the output of the channel and comparing the input and output states one can determine what is a specific action of the channel under consideration. (2) The second option is to use a collection of linearly independent test states (forming a basis of the vector space of all hermitian operators) [5-8]. By performing a correlation measurement between a specific input and corresponding output state (that has been tomographically reconstructed) we can determine the map that characterize the quantum channel. For a total determination of the map we have to use a complete set of test states.

Reconstruction of quantum channels using entangled states might seem to be more efficient since a preparation of just one state of an entangled pair is required. Nevertheless, there are two technical problems that make this approach less practical than a utilization of single particle states. Specifically, one has to generate input bi-partite entangled states with a very high fidelity (it is essential that for a reliable channel reconstruction the input test states have to be prepared with a very high fidelity). Simultaneously, the tomographic reconstruction of a bipar- tite entangled state at the output of the channel has to be almost perfect. But the most difficult obstacle is to secure that the "reference" particle from the entangled pair does not undergo uncontrolled changes during the time when the second particle is affected by the action of the quantum channel. These conditions are rather difficult to met. For this reason in the present paper we will concentrate our attention on the second scenario. This approach is based on the fact that the action of any channel is described by a linear map $\mathcal{E}$ and therefore it is completely determined by its action on basis elements, i.e. a set of linearly independent states, which play the role of test states. We assume, that these single-partite test states are known (i.e., their preparation is under a complete control). Thus the process of the channel reconstruction reduces to the reconstructions of single-particle states at the output of the quantum channel. The number of test states equals $d^{2}$, where $d$ is the dimension of the Hilbert space of a quantum system under consideration. In order to fully characterize the action of the quantum channel acting on a such quantum system we need $d^{2}\left(d^{2}-1\right)$ real parameters, i.e. in the case of a qubit channel we need 12 real parameters.

In our previous paper [9] we have analyzed the question of the process reconstruction from incomplete experimental data. That is, we have considered a situation when the correlation input-output measurements do not allow for a unique determination of the $d^{2}\left(d^{2}-1\right)$ real parameters. We have shown how to proceed with the process estimation in the case of incomplete experimental data. In this paper we want to study more delicate problem how to perform process estimation when the experimental data are incomplete and/or they are not consistent. That is, the straightforward estimation leads to maps that are not physical (i.e., they are positive but not completely positive) [10]. We will present a regularization procedure that allows us to handle such situations. Moreover, we will show that this procedure also allow us to determine optimal approximations of non-physical operations. As an example we will analyze in detail the so called universal NOT gate. The paper is organized as follows. In Section 2 we briefly describe properties of qubit 
state space while in Section 3 we discuss the structure of space of qubit operations (channels). Sections 4 and 5 are devoted to the reconstruction of quantum channels from incomplete data. Approximations of non-physical operations are derived in Section 6. The conclusions are presented in Section 7.

\section{STRUCTURE OF QUBIT STATE SPACE}

Firstly, let us consider a simple geometrical representation of a qubit state space. This state space has a topology of a sphere that is often called as the Bloch sphere. The set of all hermitian operators form a real vector space endowed with a scalar product defined by the relation $(A \mid B)=\operatorname{Tr} A B$. Consequently, any operator can be written as a linear combination of operators that form an orthogonal basis. The set of the Pauli $\sigma$-matrices, i.e. $\left\{I, \sigma_{x}, \sigma_{y}, \sigma_{z}\right\} \equiv\{I, \vec{\sigma}\}$, represents a standard choice of the operator basis for a qubit (see, e.g., Ref. [8]). The state space of a qubit is a subset of all hermitian operators with a unit trace. Except the operator $\sigma_{0}=I$ all other members of the $\sigma$-basis are traceless. Therefore any operator with a unit trace can be written as $\varrho=\frac{1}{2}(I+\vec{r} \cdot \vec{\sigma})$. Such operators represent a quantum state only when the operator $\varrho$ is positive, i.e. $|\vec{r}| \leq 1$. In this way we obtain the Bloch sphere representation of qubit states, $\varrho \leftrightarrow \vec{r}$. A state reconstruction is a task of experimental specification of the vector $\vec{r}=(x, y, z)$. ¿From the orthogonality condition $\operatorname{Tr} \sigma_{k} \sigma_{l}=2 \delta_{k l}$ we find that components of the vector $\vec{r}$ are determined by an expression $\vec{r}_{k}=\operatorname{Tr} \varrho \vec{\sigma}_{k}$. That is, they are equal to mean values of the hermitian operators (measurements) $\sigma_{x}, \sigma_{y}, \sigma_{z}$. So the complete reconstruction is straightforward: All one has to do is to measure mean values of three system operators $\sigma_{x}, \sigma_{y}, \sigma_{z}$.

Let us note that sometimes the reconstructed density operator may not satisfy the condition $|\vec{r}| \leq 1$. This failure of the reconstruction scheme is usually caused by an inconsistent measurement statistics which results in an incorrect identification of probabilities and consequently in the derivation of false mean values. The easiest way how to perform a regularization of the reconstruction in this case is the following one: The reconstructed density operator has to have always a unit trace, i.e. the operator is represented by a vector $\vec{r}$ though it might have a length larger than unity. In this case the reconstruction procedure fails since the estimated operator is not physical. One can argue that an actual physical state is the closest one to the "reconstructed" operator represented by a point on the Bloch sphere (a pure state) with $\vec{r}_{c}$ pointing in the same direction as the reconstructed vector $\vec{r}$. Formally the regularization corresponds to a multiplication of the original vector $\vec{r}$ by a positive constant $k$, i.e. $\vec{r}_{c}=k \vec{r}$. From the physical point of view this regularization can be understood as an admixture of a "white" noise described by the operator $\frac{1}{2} I$ [that is represented by the center of the Bloch sphere, i.e. $\overrightarrow{0}=(0,0,0)]$ to the measured data. Formally this "regularization" procedure reads

$$
\varrho_{c}=k \varrho+(1-k) \frac{1}{2} I=\frac{1}{2}(I+k \vec{r} \cdot \vec{\sigma}) .
$$

Such correction corresponds to the addition of completely random and equally distributed events ("clicks") to the outcome statistics of measurement results. In what follows we will utilize an analogue of this intuitive picture to regularize reconstructions of maps describing quantum channels.

\section{STRUCTURE OF QUBIT CHANNELS}

The structure of qubit channels is known mainly due to work of M.B.Ruskai et al. [12]. Let us briefly summarize main properties of qubit channels. Any completely positive map $\mathcal{E}$ can be imagined as an affine transformation of the vector $\vec{r}$, i.e. $\vec{r} \rightarrow \vec{r}^{\prime}=T \vec{r}+\vec{t}$, where $T$ is a real $3 \times 3$ matrix and $\vec{t}$ is a translation. However, this form guarantees only the preservation of a trace and the hermiticity of the transformation $\mathcal{E}$. In fact, the set of all completely positive tracepreserving maps forms a specific convex subset of all affine transformations. For qubits the number of parameters specifying the channel equals to 12 . Because of the affinity of any evolution map $\mathcal{E}$, one can use the following matrix representation

$$
\mathcal{E}=\left(\begin{array}{cc}
1 & \overrightarrow{0} \\
\vec{t} & T
\end{array}\right), \text { and } \varrho=\left(\begin{array}{c}
1 \\
\vec{r}
\end{array}\right)
$$

The coefficients of the matrix $\mathcal{E}$ are given by a relation $\mathcal{E}_{k l}=\operatorname{Tr}\left(\sigma_{k} \mathcal{E}\left[\sigma_{l}\right]\right)$, where $\sigma_{k(l)}$ are Pauli $\sigma$ matrices.

Any matrix $T$ can be written in the so-called singular value decomposition, i.e. $T=R_{U} D R_{V}$ where $R_{U}, R_{V}$ are orthogonal rotations and $D=\operatorname{diag}\left\{\lambda_{1}, \lambda_{2}, \lambda_{3}\right\}$ is a diagonal matrix with $\lambda_{k}$ being the singular values of $T$. Each three-dimensional orthogonal rotation $R_{U}$ (element of the group $\mathrm{S} 0(3))$ is related to some qubit unitary transformation $U$ (an element of the group $\mathrm{SU}(2)$ ) via the relation $U \varrho U^{\dagger}=\frac{1}{2}\left(I+\left(R_{U} \vec{r}\right) \cdot \vec{\sigma}\right)$. This means that any map $\mathcal{E}$ is a member of less-parametric family of maps of the "diagonal form" $\Phi_{\mathcal{E}}$. In particular, $\mathcal{E}[\varrho]=U \Phi_{\mathcal{E}}\left[V^{\dagger} \varrho V\right] U^{\dagger}$ where $U, V$ are unitary operators. This reduction of parameters is very helpful, and most of the properties (also complete positivity) of $\mathcal{E}$ is reflected by the properties of $\Phi_{\mathcal{E}}$. The map $\mathcal{E}$ is completely positive (CP) only if $\Phi_{\mathcal{E}}$ is also CP. Let us note that $\Phi_{\mathcal{E}}$ is determined not only by the matrix $D$, but also by a new translation vector $\vec{\tau}=R_{U} \vec{t}$, i.e. under the action of the map $\Phi_{\mathcal{E}}$ the Bloch sphere transforms as follows $r_{j} \rightarrow r_{j}^{\prime}=\lambda_{j} r_{j}+\tau_{j}$. 


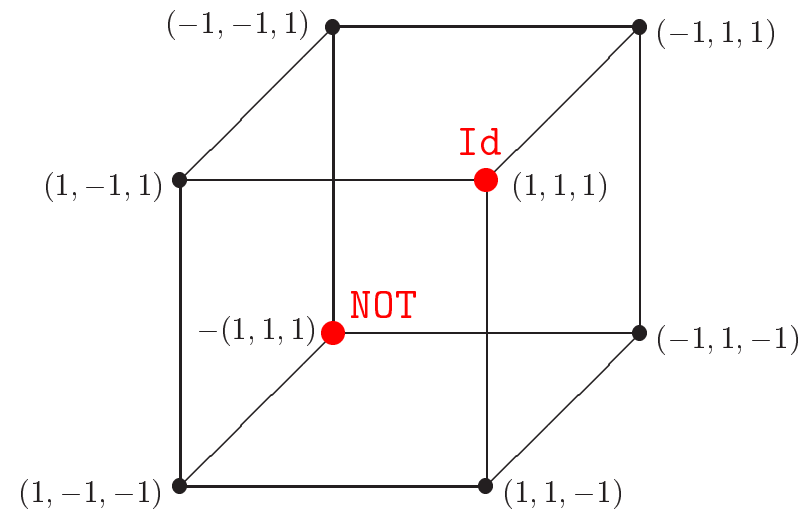

FIG. 1. The set of positive unital trace-preserving linear maps $\Phi_{\mathcal{E}}$ parametrized by three real parameters $\lambda_{1}, \lambda_{2}, \lambda_{3}$. The point $\lambda_{1}=\lambda_{2}=\lambda_{3}=-1$ corresponds to a physically unrealizable transformation - the universal NOT (denoted as NOT in the figure) operation, i.e. The point $\lambda_{1}=\lambda_{2}=\lambda_{3}=1$ represents the identity map $\mathcal{I}$ (denoted as Id)

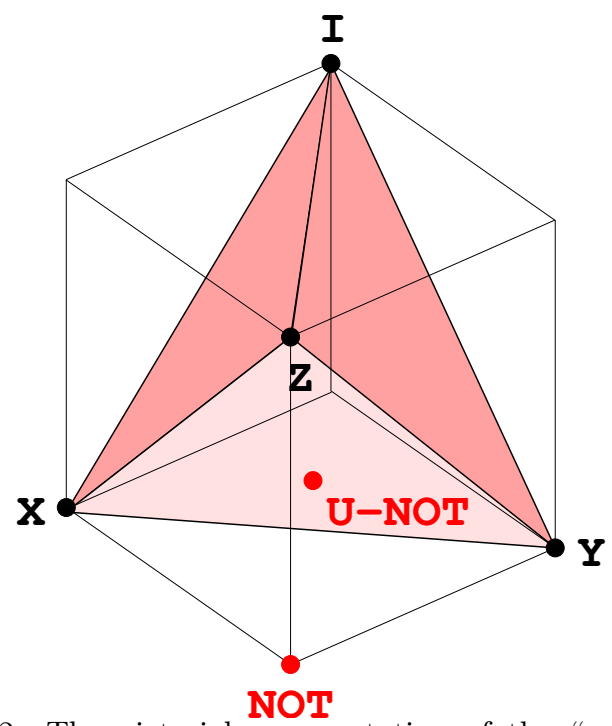

FIG. 2. The pictorial representation of the "position" of completely positive unital maps in the space of all positive unital maps. The CP unital maps form a tetrahedron with four unitary transformations in its vertices (extremal points), that correspond to $\sigma$-matrices. The un-physical universal NOT operation (denoted as NOT) and its best completely positive approximation called the optimal universal NOT (denoted as U-NOT) are shown as well. The optimal universal $\mathrm{NOT}$ is represented by the point $\lambda_{1}=\lambda_{2}=\lambda_{3}=-1 / 3$.

A special type of completely positive maps is a class of unital transformations, i.e. maps for which the total mixture (the center of the Bloch sphere) is not affected by the transformation. It means that the translation term vanishes, i.e $\vec{t}=\vec{\tau}=\overrightarrow{0}$. In this case the geometrical analysis is quite simple. The positivity of the transformation $\Phi_{\mathcal{E}}$ corresponds to the conditions $\left|\lambda_{k}\right| \leq 1$, i.e. these transformations are represented by points lying in- side a cube (see Fig.1). The conditions of the complete positivity [12] requires the validity of the following four inequalities

$$
\left|\lambda_{1} \pm \lambda_{2}\right| \leq\left|1 \pm \lambda_{3}\right|
$$

These inequalities specify a tetrahedron inside a cube of all positive unital maps with the extreme points being four unitary transformations $I, \sigma_{x}, \sigma_{y}, \sigma_{z}$ (see Fig.2). As a result of this analysis one can conclude that the unital completely positive maps $\Phi_{\mathcal{E}}$ form a tetrahedron.

\section{THE CHANNEL RECONSTRUCTION: INCONSISTENT STATISTICS}

Let us consider a situation when we want to estimate a channel, but neither experimental data nor non-trivial prior knowledge are available. Following the approach utilized for quantum state estimation [15] one can assume that in the absence of knowledge about the character of quantum channel the most reliable estimation of this channel corresponds to an equally weighted "average" over all possible quantum maps. The question is what is the average over all completely positive maps? We have already argued [9] that for qubits this average is the map $\mathcal{A}$ that transforms the whole state space into the total mixture, i.e. $\mathcal{A}[\varrho]=\frac{1}{2} I$. The reasoning goes as follows: The maps $\mathcal{E}_{ \pm} \leftrightarrow(T, \pm \vec{t})$ have the property that if one of them is completely positive the second one is $\mathrm{CP}$ as well. Consequently, the average $\frac{1}{2}\left(\mathcal{E}_{+}+\mathcal{E}_{-}\right)$is a unital map, i.e. the average of all maps will be a unital map. As we already said, the unital maps (up to unitary transformations) form a tetrahedron. The average over all points in tetrahedron is represented by the center of the tetrahedron, i.e. $\lambda_{1}=\lambda_{2}=\lambda_{3}=0$, which corresponds to the contraction into the total mixture. Therefore, the average over all completely positive maps of acting on a qubit is the contraction of the whole Bloch sphere into its center, i.e. $\mathcal{A}[\varrho]=\frac{1}{2} I$.

The result of a complete reconstruction based on the four test states (i.e., any collection of four mutually linearly independent qubit states) is a map that is for sure trace-preserving and positive. However, it could happen that it is not completely positive. How to extract the physical map $\mathcal{E}_{c}$ from an unphysical result $\mathcal{E}$ ? One way is to follow a similar reasoning like for the state reconstruction, when an unphysical result was corrected (regularized) by adding a noise into the system.

When the reconstructed map is not completely positive we can regularize this result by an analogue of the total mixture, i.e. the map $\mathcal{A}$. In particular, this regularization of a quantum channel reads

$$
\mathcal{E}_{c}=k \mathcal{E}+(1-k) \mathcal{A}=\left(\begin{array}{cc}
1 & \overrightarrow{0} \\
k \vec{t} & k T
\end{array}\right) .
$$


The correction (regularization) corresponds to a "minimal" adjustment of the parameter $k$ such that the map $\mathcal{E}_{c}$ is completely positive.

Let us estimate what is this minimal (critical) value of the parameter $k$, i.e. the value which surely regularizes any positive map and transforms it into a CP map. Trivially, it is enough to set $k=0$. In this case we completely ignore the measured data and the "corrected" map is $\mathcal{A}$. However, we are interested in some nontrivial bound, i.e. in the largest possible value of $k$. As we have already mentioned, the reconstructed map is always positive. Consider, for simplicity, that the map is also unital. Then the "worst" example of a positive map, which is not completely positive, is the universal NOT operation. In this case the distance between this map and the tetrahedron of completely positive maps is extremal (see Fig.2). This artificial example serves as a good test of our method, and gives us some bound on $k$, i.e. a value that surely corrects each result. The conditions of the complete positivity given in Eq.(3) imply that $k=1 / 3$, i.e. $\lambda_{1}=\lambda_{2}=\lambda_{3}=-1 / 3$ (see Fig.2). Surprisingly, this is the same result as the one that has been obtained in Ref. [13] where the best (optimal) completely positive approximation of the universal NOT operation, i.e. an optimal universal NOT machine has been presented. In this sense our correction method works optimally.

\section{THE CHANNEL RECONSTRUCTION: INCOMPLETE DATA}

In this section we will present the reconstruction scheme which can be used when the number of test states is reduced so that the complete reconstruction of the channel cannot be performed [9]. On the other hand we assume that each of the state used is represented by an infinite ensemble of identically prepared states so the complete tomography of a corresponding state at the output of the channel can be performed.

In the case of qubit channels the aim is to perform the reconstruction based on $n=0,1,2,3,4$ measured inputoutput correlations of the form $\varrho_{j} \rightarrow \varrho_{j}^{\prime}$. We have already shown that having no information $(n=0)$, the best estimation of the map is the contraction into a total mixture (an average over all quantum channels) i.e. $\mathcal{E}_{0}=\mathcal{A}$. Motivated by such result, the reconstruction strategy (see Ref. [9]) is as follows: All undetermined states (belonging to the complement of the linear span of the used input states) are assumed to be transformed into the total mixture. That is, if a given test state is not explicitly used for a channel reconstruction (i.e., it is not know how this state is transformed by the action of the channel) it is assumed that the channel transforms this state into a total mixture. This additional assumption complements the knowledge of how other test states are actually transformed by the channel and allows us to use the deterministic procedure of channel reconstruction (for details see e.g. Refs. [6,5,7]).
Because of the ad hoc assumption about the transformation of unused test states it might happen that the resulting map is not completely positive. In this case the estimation procedure has to be complemented by a search for a map for which the total mixture is "shifted" as little as possible, i.e. the estimated channel is preferably unital. A specific situation occurs when the data contain information about the transformation of the total mixture. In this case the strategy suggests to use the state $\mathcal{E}\left[\frac{1}{2} I\right]$ as the state on which all other undetermined states are mapped. As before, if such map is not completely positive we have to search for a map, for which the deviation from the transformation $\mathcal{E}\left[\frac{1}{2} I\right]$ is minimal.

The method described above is discussed in detail in Ref. [9]. We note that this (incomplete) reconstruction can also fail (it gives no result), because it can happen that no physical channel is is compatible with given experimental data. Even if the reconstructed operators $\varrho_{j}^{\prime}$ describe valid quantum states, the incomplete data can be in a contradiction with the condition of a complete positivity. In what follows we will briefly describe our strategy on a particular example - estimation of the identity channel (i.e. the channel, that does not change input states at all). For more detailed and more general description of this strategy see Ref. [9].

\section{A. Case study: identity channel}

In what follows we will perform a step-by-step reconstruction of a qubit channel based on a knowledge of how a single, two and three test states are transformed under the action of a given channel.

Single test state. Let us assume that our knowledge about the action of a particular channel is represented by the assignment

$$
\varrho_{1} \rightarrow \varrho_{1}^{\prime}=\varrho_{1}
$$

We remind us that each state can be written in the form $\varrho_{1}=\frac{1}{2}(I+\vec{r} \cdot \vec{\sigma})=\frac{1}{2}\left(I+w S_{z}\right)$, where $S_{z}=$ $|\psi\rangle\left\langle\psi|-| \psi_{\perp}\right\rangle\left\langle\psi_{\perp}\right|$ with $|\psi\rangle,\left|\psi_{\perp}\right\rangle$ being eigenvectors of the operator $\varrho_{1}$ and $w$ describes an impurity of the state under consideration, i.e. $w=\sqrt{1-2 \operatorname{Tr} \varrho_{1}^{2}}$. If $w=0$ then $\varrho_{1}$ describes maximally mixed state and for $w=1$ the state is pure. In the Bloch-sphere picture the parameter $w$ corresponds to the distance between the total mixture (the center of the sphere) and a point corresponding to the given state. One can define a new operator basis $S_{x}, S_{y}, S_{z}$ such that $S_{z}$ is given as before, and $S_{j}=U \sigma_{j} U^{\dagger}$ with unitary $U$. In this new basis the action of the channel is described by the matrix

$$
\mathcal{E}_{1}=\left(\begin{array}{cccc}
1 & 0 & 0 & 0 \\
x & a & d & 0 \\
y & b & e & 0 \\
z & c & f & 1
\end{array}\right)
$$


and the task of the estimation is to specify all matrix elements. Our strategy suggests that all states (belonging to the complement of the linear span of $\varrho_{1}$ ) are transformed into the total mixture, i.e. we set all the parameters equal to zero.

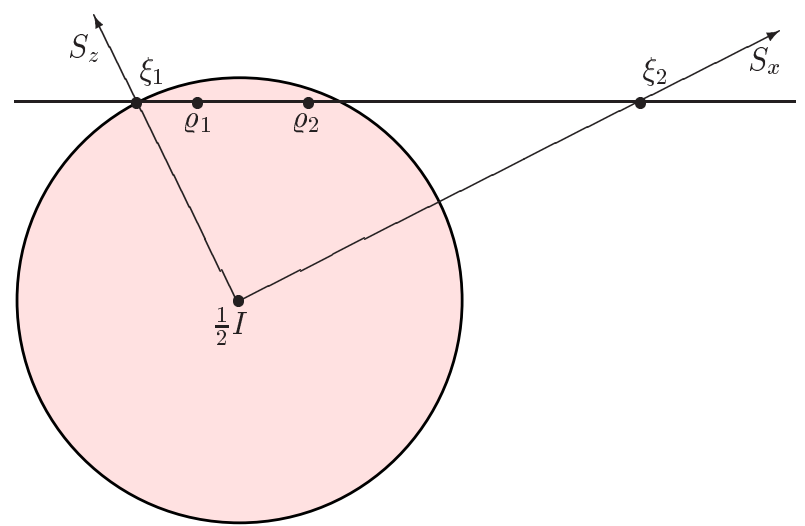

FIG. 3. The pictorial description of the channel estimation when two states $\rho_{1}$ and $\rho_{2}$ are used to test the action of a quantum channel and they are transformed according to Eq. (7).

Two test states. In this case the knowledge about the action of the channel is represented by a transformation of two test state given by the following relations

$$
\varrho_{1} \rightarrow \varrho_{1}^{\prime}=\varrho_{1} ; \quad \varrho_{2} \rightarrow \varrho_{2}^{\prime}=\varrho_{2} .
$$

Irrespective of states $\varrho_{1}$ and $\varrho_{2}$ we use, they specify a line crossing the Bloch sphere. In particular, from Eq. (7) we obtain a knowledge about the transformation of all states of the form

$$
\varrho_{\lambda}=\lambda \varrho_{1}+(1-\lambda) \varrho_{2}
$$

with a real parameter $\lambda$. This line crosses the Bloch sphere in two extremal points representing pure states. Let us choose one of these pure states [denoted by $\xi_{1}=$ $\left.\frac{1}{2}\left(I+w S_{z}\right)\right]$. We also define a new operator (not necessarily positive) $\xi_{2}=\frac{1}{2}\left(I+v S_{x}\right)$, which in the Bloch-sphere picture corresponds to the intersection of the line $\varrho_{\lambda}$ and the line orthogonal at the center of the Bloch sphere to the line given by points $\left\{\xi_{1}, \frac{1}{2} I\right\}$ (see Fig.3). This new state $\xi_{2}$ defines the operator $S_{x}$. Using the new operator basis $S_{x}, S_{y}, S_{z}$ we can express the map characterizing the action of the channel as

$$
\mathcal{E}_{2}=\left(\begin{array}{cccc}
1 & 0 & 0 & 0 \\
x & 1 & a & 0 \\
y & 0 & b & 0 \\
z & 0 & c & 1
\end{array}\right)
$$

We again set all free (unspecified by the measurement) parameters to zero. As a result we find that the transformation $\mathcal{E}_{2}=\operatorname{diag}\{1,1,0,1\}$ is not completely positive, i.e. it does not describe a valid quantum channel. To preserve the unitality of the channel $\left(\mathcal{E}_{2}\left[\frac{1}{2} I\right]=\frac{1}{2} I\right)$ one has to verify the complete positivity of the transformation $\mathcal{E}_{2}=\operatorname{diag}\{1,1, k, 1\}$. We find out that the only possibility is to take $k=1$. Consequently, the identity channel is correctly estimated, but we are still not sure, whether the map is unital. However, we have to note one specific case, when the total mixture is among the states $\varrho_{\lambda}$, i.e. $\xi_{2}=\frac{1}{2} I$. In this case

$$
\mathcal{E}_{2}=\left(\begin{array}{cccc}
1 & 0 & 0 & 0 \\
0 & e & a & 0 \\
0 & f & b & 0 \\
0 & g & c & 1
\end{array}\right)
$$

and our reconstruction procedure leads us to the channel $\mathcal{E}_{2}=\operatorname{diag}\{1,0,0,1\}$, i.e. the values of all free parameters are equal to zero. Note that in this case $\mathcal{E}_{2}=\mathcal{E}_{1}$.

Three test states. Let us consider that the linear span of $\varrho_{1}, \varrho_{2}, \varrho_{3}$ does not contain the total mixture. In this case the estimated transformation takes the form

$$
\mathcal{E}_{3}=\left(\begin{array}{cccc}
1 & 0 & 0 & 0 \\
x & 1 & 0 & 0 \\
y & 0 & 1 & 0 \\
z & 0 & 0 & 1
\end{array}\right)
$$

The only possibility to preserve the complete positivity of $\mathcal{E}_{3}$ is to chose $x=y=z=0$, which is completely compatible with our strategy. Consequently, the channel is estimated perfectly and it is described by the transformation $\mathcal{E}_{3}=\operatorname{diag}\{1,1,1,1\}=\mathcal{I}$. In the case, when from the measured data it follows that $\frac{1}{2} I \rightarrow \frac{1}{2} I$, the estimation coincides with the reconstruction with two test states, where the transformation of the total mixture is estimated to be $\mathcal{E}\left[\frac{1}{2} I\right]=\frac{1}{2} I$. The only difference is that in the three-state case the unitality is guaranteed by the data. The reconstruction gives us the same result in both cases, i.e. $\mathcal{E}_{3}=\mathcal{I}$. As a result we find that identity channels for qubits can be uniquely identified using just three test states.

Let us summarize the incomplete reconstruction of the identity channel. The hierarchy of estimations on different levels specified by the number of test states is as follows

$$
\begin{aligned}
& \mathcal{E}_{0}=\mathcal{A} ; \\
& \mathcal{E}_{1}=\operatorname{diag}\{1,0,0,1\} ; \\
& \mathcal{E}_{2}=\mathcal{E}_{3}=\mathcal{E}_{4}=\mathcal{I} .
\end{aligned}
$$

¿From our previous discussion we can conclude that the identity channel can be reconstructed using just three test states. Given the fact, that for any unitary channel $\mathcal{E}$ the induced map $\Phi_{\mathcal{E}}$ represents the identity channel, i.e. $\Phi_{\mathcal{E}}=\mathcal{I}$ we can conclude that for a complete determination of a unitary channel we need just three test states. A unitary transformation is determined by the choice of the basis $S_{x}, S_{y}, S_{z}$. 


\section{COMBINATION OF DATA IMPERFECTIONS: INCOMPLETE AND INCONSISTENT DATA}

In this section we will study how to perform a process reconstruction when the data obtained from the measurement are incomplete as well as inconsistent. To secure that the incomplete reconstruction does not fail we can adopt the regularization procedure as described earlier in the paper. In particular, we have two options: (i) either to regularize the output test states, or (ii) to regularize the estimated map itself.

The first scenario has to be used always when the state reconstruction of some of the output test states $\varrho_{j}^{\prime}$ fails. However, also if all the test states are estimated (reconstructed) correctly (i.e. they are legitimate physical states), the complete positivity is not guaranteed and the process reconstruction might fail. This situation can occur even for two test states. Specifically, the assignments $\varrho_{1} \rightarrow \varrho_{1}^{\prime}$ and $\varrho_{2} \rightarrow \varrho_{2}^{\prime}$ are compatible with some completely positive map $\mathcal{E}$ if and only if $D\left(\varrho_{1}, t \varrho_{2}\right) \geq D\left(\varrho_{1}^{\prime}, t \varrho_{2}^{\prime}\right)$ for all real positive $t$ [14]. Unfortunately, for three and four test states no similar result is known. Therefore the complete positivity of the process reconstruction has to be checked for each individual case separately.

In what follows we will utilize (demonstrate) two reconstruction strategies:

- We will use the reconstruction from incomplete data as discussed above. In the case of a failure (the reconstructed channel is not a CP map) a regularization of outputs of test states is performed (noise is admixed into the output states, so that the reconstructed map becomes $\mathrm{CP}$ ).

- We will assume that an unknown map transforms all states except the basis test states into the total mixture, i.e. we set all free parameters to zero. If the resulting map is not completely positive, then the map will be regularized by adding the average channel $\mathcal{A}$.

Let us consider a particular example that allows us to demonstrate these two methods. In our example we will use an "artificial" and unphysical data generated by the universal NOT operation [13]. The logical NOT operation is defined by relations $|0\rangle \rightarrow|1\rangle$, $|1\rangle \rightarrow|0\rangle$ in a computer basis $\{|0\rangle ;|1\rangle\}$. These relations do not completely determine a quantum channel, i.e. many quantum channels perform such transformations. For instance, the "classical" NOT can be viewed as the transformation of the Bloch sphere into the line connecting North $(|1\rangle\langle 1|)$ and South Pole $(|0\rangle\langle 0|)$, i.e. $\operatorname{NOT}_{c}[\varrho]=\frac{1}{2}\left(I+\left\langle\sigma_{z}\right\rangle_{\varrho} S_{z}\right)$ with $\left\langle\sigma_{z}\right\rangle_{\varrho}=\operatorname{Tr} \varrho \sigma_{z}$. There exists also a unitary ("quantum") realization of the logical NOT operation, i.e. $\mathrm{NOT}_{q}[\varrho]=\sigma_{x} \varrho \sigma_{x}$. However, a natural generalization of the NOT operation is the so called universal NOT gate, which performs the transformation $|\psi\rangle \rightarrow\left|\psi_{\perp}\right\rangle$ for all states $|\psi\rangle$. The universal NOT gate is not completely positive (for mixed states we will use the notation $\varrho \rightarrow \varrho^{T}$ ). The optimal universal NOT operation is the closest physically valid map that performs approximatively the universal NOT. In Ref. [13] it has been shown that the corresponding quantum channel that maximizes the average fidelity under given constraints reads $\mathcal{E}_{\mathrm{NOT}}=\operatorname{diag}\{1,-1 / 3,-1 / 3,-1 / 3\}$.

\section{A. Case study: the universal NOT gate}

Formally the universal NOT operation determines the following transformations on a set of test (basis) states

$$
\begin{gathered}
\varrho_{x}=\frac{1}{2}\left(I+\sigma_{x}\right) \rightarrow \varrho_{x}^{\prime}=\frac{1}{2}\left(I-\sigma_{x}\right) ; \\
\varrho_{y}=\frac{1}{2}\left(I+\sigma_{y}\right) \rightarrow \varrho_{y}^{\prime}=\frac{1}{2}\left(I-\sigma_{y}\right) ; \\
\varrho_{z}=\frac{1}{2}\left(I+\sigma_{z}\right) \rightarrow \varrho_{z}^{\prime}=\frac{1}{2}\left(I-\sigma_{z}\right) ; \\
\varrho_{0}=\frac{1}{2} I \rightarrow \varrho_{0}^{\prime}=\frac{1}{2} I .
\end{gathered}
$$

Let us start with the first reconstruction strategy as described above. In the case when just one or two states have been used to test the action of the quantum channel no regularization is needed to estimate the channel.

(1) When we use only a single test state (e.g., a pure state) the resulting estimated map is a contraction of the Bloch sphere into a line connecting two mutually orthogonal states. If we use the data $\varrho_{z} \rightarrow \varrho_{z}^{\prime}$, then this line is given by the points $(0,0, \pm 1)$, i.e. the Bloch sphere is mapped into the $z$ axis. This map can be understand as the "classical" logical NOT.

(2) In the case of two test states, we obtain a unitary rotation. If we use $\varrho_{z} \rightarrow \varrho_{z}^{\prime}$ and $\varrho_{y} \rightarrow \varrho_{y}^{\prime}$, then the result is a rotation by the angle $\pi$ around the $x$ axis, i.e. the $\sigma_{x}$ operation. This operation is usually referred to as the "quantum" (unitary) logical NOT (see Fig.4).

When three test states are used to determine the action of the universal NOT gate we face a serious difficulty: Let us consider transformations of three test states $\varrho_{x, y, z} \rightarrow \varrho_{x, y, z}^{\prime}$. In this case no completely positive map exists. Therefore, a regularization of output states is required - in particular, we have to determine a minimal amount of noise that is "included" in the output states so that the channel estimation with these regularized (noisy) output states will result in a CP map. It turns out that the amount of noise corresponds to the value $k=1 / 3$. Therefore the transformation that is closest to the universal NOT gate and is CP has the form

$$
\varrho_{j}^{\prime}=\frac{1}{3} \varrho_{j}^{T}+\frac{2}{3} \frac{1}{2} I .
$$

We can conclude that already for three test states we find that the reconstructed map that is $\mathrm{CP}$ is the optimal universal NOT operation [13]. In Fig.4 we see how 
the estimation of the map changes with the number $n$ of used test states.

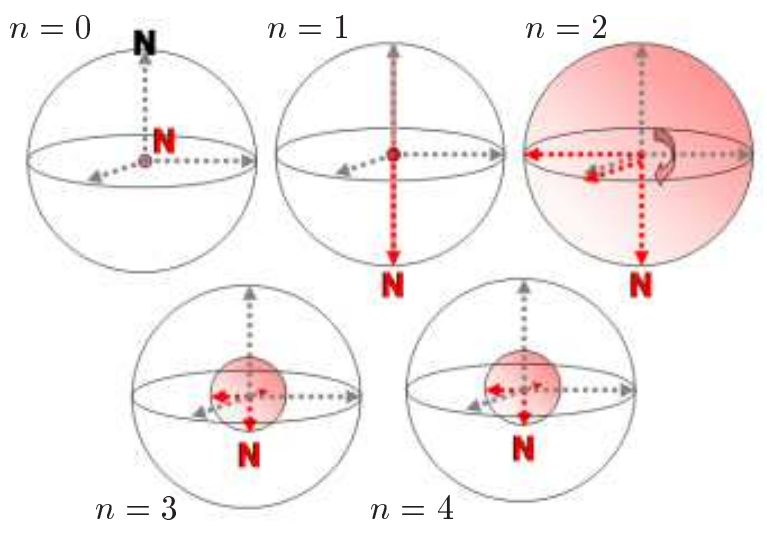

FIG. 4. The figure represents how our knowledge about the channel (the universal NOT gate) is improved with increasing the number of test states when the first reconstruction strategy is used. If no measurement has been performed then the best possible estimation of the channel is the contraction of the Bloch sphere into its center $(n=0)$. When a single test state $\varrho_{z} \rightarrow \varrho_{z}^{\prime}$ is used the optimal estimation of the transformation is a contraction of the whole Bloch sphere into a line connecting the "north" (denoted as $N$ ) and "south" poles of the Bloch sphere (i.e. the points $(0,0, \pm 1)$, respectively). This contraction is represented by the figure $n=1$ in the picture. When two test states $(n=2)$ are used, i.e. $\varrho_{z} \rightarrow \varrho_{z}^{\prime}$ and $\varrho_{y} \rightarrow \varrho_{y}^{\prime}$, then the result of the estimation of the action of the quantum channel is a rotation by the angle $\pi$ around the $x$ axis, i.e. the $\sigma_{x}$ operation. Finally, when three $(n=3)$ or four $(n=4)$ test states are used to determine the action of the quantum channel prescribed by the universal NOT transformation, the best estimation of the channel is the optimal universal NOT gate (see the two figures of the lower line in the picture, both figures are the same and they describe the action of the optimal universal NOT gate). In this case the regularization of the output test states has been used in order to estimate a map that is completely positive. As a specific example we show how the "north" pole $\mathrm{N}$ of the Bloch sphere is transformed under the action of estimated maps.

In what follows we will study the second strategy for a reconstruction (estimation) of the best possible approximation of the universal NOT gate. Instead of searching for a completely positive map by "admixing" the smallest possible amount of noise into the outputs of the test states, let us assume that all states except the test states are mapped into the total mixture ${ }^{1}$. Using this approach we can be sure that the resulting map is positive, but not completely positive. Therefore we have to use a regularization of the map. Obviously, in this case the resulting map will not explicitly satisfy conditions imposed by the transformation of test states, $\varrho_{j} \rightarrow \varrho_{j}^{\prime}$, i.e. with the given data. But this has to be expected since it is the only way how to impose the $\mathrm{CP}$ condition on the reconstructed map. Using this type of regularization of the map we arrive at the same estimations as in the case of previous strategy. However, for $n=2$ (two test states) the situation is different. In this case, the reconstructed map acts as follows

$$
\vec{r}=(x, y, z) \rightarrow \vec{r}^{\prime}=(0,-y,-z) .
$$

That is, the states of the form $\varrho=\frac{1}{2}\left(I+x \sigma_{x}\right)$ are transformed into the total mixture. Consequently, the map is unital and has a diagonal form with $\lambda_{1}=0, \lambda_{2}=\lambda_{3}=$ -1 , which is not compatible with the complete positivity (see Fig.2). The parameter $k=1 / 2$ can be used to correct this map. This value of $k$ can be derived from the inequalities $\left|\lambda_{1} \pm \lambda_{2}\right| \leq\left|1 \pm \lambda_{3}\right|$. One can observe this result in Fig. 2 as the closest point from the tetrahedron to the point $(0,-1,-1)$. The whole incomplete reconstruction using this method is depicted in Fig.5. In Tab. 1 we present diagonal elements (all other matrix elements are equal to zero) of matrices corresponding to reconstructed maps based on the results of measurement of $n$ test states. We present results for both reconstruction methods.

\begin{tabular}{|l||c|c|}
\hline \hline & 1st method & 2nd method \\
\hline $\mathcal{E}_{0}$ & $\{1,0,0,0\}$ & $\{1,0,0,0\}$ \\
$\mathcal{E}_{1}$ & $\{1,0,0,1\}$ & $\{1,0,0,1\}$ \\
$\mathcal{E}_{2}$ & $\{1,1,-1,-1\}$ & $\{1,0,-1 / 2,-1 / 2\}$ \\
$\mathcal{E}_{3}$ & $\{1,-1 / 3,-1 / 3,-1 / 3\}$ & $\{1,-1 / 3,-1 / 3,-1 / 3\}$ \\
$\mathcal{E}_{4}$ & $\{1,-1 / 3,-1 / 3,-1 / 3\}$ & $\{1,-1 / 3,-1 / 3,-1 / 3\}$ \\
\hline \hline
\end{tabular}

TABLE I. The two reconstruction scenarios for an un-physical universal NOT gate result in a sequence of maps depending on the number of test states. The subscript in the description of a given map $\mathcal{E}_{j}$ corresponds to the number of test states that have been used in the reconstruction. From the table it is clear, that both methods give us the same process estimation except for the case when two test states have been used.

\footnotetext{
${ }^{1}$ More specifically, states that are complemented to a linear span of the test states.
} 


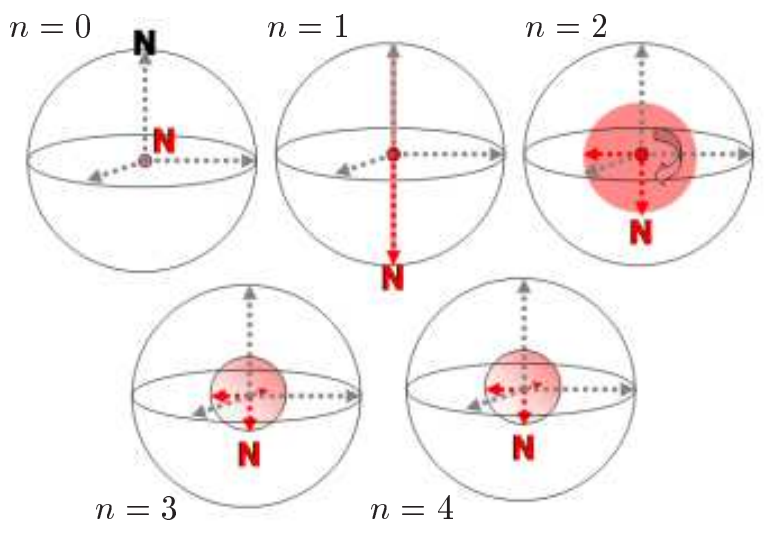

FIG. 5. The figure represents how our knowledge about the channel (the universal NOT gate) is improved with increasing the number of test states when the second reconstruction strategy is used. If no measurement has been performed then the best possible estimation of the channel is the contraction of the Bloch sphere into its center $(n=0)$. When a single test state $\varrho_{z} \rightarrow \varrho_{z}^{\prime}$ is used the optimal estimation of the transformation is a contraction of the whole Bloch sphere into a line connecting the "north" and "south" poles of the Bloch sphere (i.e. the points $(0,0, \pm 1)$, respectively). This contraction is represented by the figure $n=1$ in the picture. When two test states $(n=2)$ are used, i.e. $\varrho_{z} \rightarrow \varrho_{z}^{\prime}$ and $\varrho_{y} \rightarrow \varrho_{y}^{\prime}$, then the result of the estimation of the action of the quantum channel is described by the transformation (19). Consequently, the map is unital and has the diagonal form with $\lambda_{1}=0, \lambda_{2}=\lambda_{3}=-1$. Unfortunately this map is not completely positive. The regularization parameter $k=1 / 2$ is used to correct this map. Finally, when three $(n=3)$ or four $(n=4)$ test states are used to determine the action of the quantum channel prescribed by the universal NOT transformation, the best estimation of the channel is the optimal universal NOT gate.

\section{CONCLUSION}

Reconstruction of quantum maps is a challenging problem motivated mainly by experimental realizations of quantum gates. These gates have to be tested thoroughly in order to use them for any quantum computation. Another important application of a channel reconstruction is in quantum communication when characteristics of a quantum channel have to be determined from a limited set of tests performed on the channel.

In the present paper we have discussed some strategies how to estimate quantum channels when only incomplete and/or incompatible data from measurements is available. The incompatibility of available data results in estimated maps that are not completely positive. In this case a regularization of the reconstruction procedure is required in order to recover a physical (CP) map.

The regularization method represents a correction of insufficient statistics. It uses a single parameter $k$ that can be understood as an addition of a white noise into our data. In principle, we can face two situations: Either reconstructions of all test states at the output of the channel correspond to proper quantum states, or there exist some outcomes that are not proper quantum states. In this case each of these states can be corrected by adding some noise (using the multiplicative factor $k$ as discussed above). We have to keep in mind that the parameter $k$ can be state dependent. Therefore, we have to choose the smallest value of $k$ in order to correct the map. Once all the outputs (as estimated from the measured data) are proper physical states we can start to reconstruct the map itself. In spite of the fact that all the test states at the output are proper physical states the map that is estimated on the basis of these states may be not completely positive. In this case we have to search for the largest $k$, for which the corrected map $\mathcal{E}_{c}=k \mathcal{E}+(1-k) \mathcal{A}$ is completely positive. We have shown that at least for unital maps the value $k=1 / 3$ always regularizes the estimated map.

In order to illustrate our methods we have used the data generated by an unphysical map - the universal NOT gate. We have shown that using both approaches the result is the same and the reconstructed map is the best (optimal) approximation of the NOT operation [13].

\section{ACKNOWLEDGMENT}

This was work supported in part by the European Union projects QUPRODIS (IST-2001-38878) and CONQUEST (MRTN-CT-2003-505089). M.Z. would like to thank for a partial support via the grant 201/04/1153 of the GACR.

[1] G.M. D'Ariano and P.Lo Presti, Tomography of quantum operations, Phys.Rev.Lett. 86, 4195 (2001).

[2] G.M.D'Ariano and P.Lo Presti, "Characterization of quantum devices" in Quantum Estimations: Theory and Experiment, Springer Series on Lecture Notes in Physics, Band 649, eds. G.M.Paris and J. Řeháček (SpringerVerlag, Berlin, 2004), p. 299.

[3] M.Ježek, J.Fiurášek, Z.Hradil, "Quantum inference of states and processes", Phys.Rev.A 68, 012305 (2003).

[4] Z. Hradil, J. Řeháček, J. Fiurášek, and M. Ježek, Maximum-Likelihood Methods in Quantum Mechanics in Quantum State Estimation, Springer Series on Lecture Notes in Physics, Band 649, eds. M.G.A. Paris and J.Řeháček (Springer-Verlag, Berlin, 2004), p. 63.

[5] J.F. Poyatos, J.I. Cirac, and P. Zoller, Complete characterization of a quantum process: two-qubit quantum gate, Phys. Rev. Lett. 78, 390 (1997). 
[6] I.L. Chuang and M.A. Nielsen, Prescription for experimental determination of the dynamics of a quantum black box, J. Mod. Opt. 44, 2455 (1997).

[7] V. Bužek, Reconstruction of Liouvillian superoperators, Phys. Rev. A 58, 1753 (1998).

[8] M.A. Nielsen and I.L. Chuang, Quantum Computation and Quantum Information (University Press, Cambridge, 2000).

[9] M. Ziman, M. Plesch, and V.Bužek, On the reconstruction of superoperators from incomplete data, to appear in Foundations of Physics, quant-ph/0406088.

[10] M. Ziman, T. Hannemann, M. Plesch, V. Bužek, and Ch. Wunderlich, in preparation

[11] M. Ziman, M. Plesch, and V. Bužek, in preparation
[12] M.B. Ruskai, S. Szarek, and E. Werner, An analysis of completely positive tracepreserving maps on $2 x 2$ matrices, Lin. Alg. Appl. 347, 159 (2002).

[13] V. Bužek, M. Hillery, and R.F. Werner, Optimal manipulation with qubits:Universal-NOT gate, Phys.Rev.A 60, R2626 (1999).

[14] A. Carlini and M. Sasaki, Geometrical conditions for completely positive trace-preserving maps and their application to a quantum repeater and a state-dependent quantum cloning machine, Phys. Rev. A 68, 042327 (2003).

[15] V. Bužek and R. Derka, Quantum Observations, in Coherence and Statistics of Photons and Atoms, ed. J. Peřina (John Wiley \& Sons, New York, 2001), p. 198. 